\title{
A pattern search method for improving the operation of sewer systems*
}

\author{
S. Leirens, J.M. Giraldo, R.R. Negenborn, and B. De Schutter
}

If you want to cite this report, please use the following reference instead:

S. Leirens, J.M. Giraldo, R.R. Negenborn, and B. De Schutter, "A pattern search method for improving the operation of sewer systems," Proceedings of the 12th IFAC Symposium on Large Scale Systems: Theory and Applications, Villeneuve d'Ascq, France, pp. 591-596, July 2010.

Delft Center for Systems and Control Delft University of Technology

Mekelweg 2, 2628 CD Delft

The Netherlands

phone: +31-15-278.24.73 (secretary)

URL: https: //www.dcsc.tudelft.nl

*This report can also be downloaded viahttps://pub.deschutter.info/abs/10_014.html 


\title{
A Pattern Search Method for Improving the Operation of Sewer Systems
}

\author{
S. Leirens ${ }^{*}$ J.M. Giraldo* R.R. Negenborn ${ }^{* *}$ B. De Schutter ${ }^{* *}$ \\ * Department of Electrical and Electronic Engineering, \\ Universidad de los Andes, Bogotá D.C., Colombia \\ (e-mail: sleirens@uniandes.edu.co,jm.giraldo129@uniandes.edu.co) \\ ** Delft Center for Systems and Control, \\ Delft University of Technology, Delft, The Netherlands \\ (e-mail: r.r.negenborn@tudelft.nl,b.deschutter@tudelft.nl)
}

\begin{abstract}
Sewer systems are large-scale systems that collect and transport stormwater and sanitary sewage out of urban areas. Sewer systems are mainly operated using pumping stations and pollutants are removed from sewage by treatment plants before water is released into the environment. When a sewer overflow occurs, e.g., caused by a strong rainfall, sewage is discharged directly into the environment without any treatment. An efficient use of storage capacities and pumping stations can help to minimize the environmental pollution caused by sewer overflows. In this paper a nonlinear predictive control approach is presented to improve the operation of sewer systems. To deal with the nonlinear and nondifferentiable features of the prediction model used, a pattern search method is proposed to solve the underlying optimization problem. The technique proposed is implemented on a part of the sewer system of Bogotá, Columbia. Simulation results illustrate the potential of the approach.
\end{abstract}

Keywords: Sewer systems, model predictive control, nonlinear optimization, pattern search.

\section{INTRODUCTION}

Sewer systems are large-scale water transportation systems that span complete urban areas. On the one hand, they are the complementary part of water distribution systems by draining wastewater (produced by domestic usage and industrial facilities) out of urban areas up to wastewater treatment plants that remove physical, chemical, and biological contaminants, before releasing the wastewater into the natural environment (Hendricks, 2005). On the other hand, sewer systems prevent stormwater arising from excessive rainfall to flood into streets by draining water up to neighboring rivers. In separated sewer systems, stormwater and wastewater are transported using two different grids, whereas in combined sewer systems they mix in the same pipes. Both types are generally present in old cities: a combined sewer system is used in the older parts and a separated sewer system is used in the more recent parts.

Clearly, the efficient operation of sewer systems is of crucial importance to minimize the amount of pollutants that are released into the environment. Cities in areas that experience heavy rainfall as well as coastal towns that regularly experience storms are hereby of major concern. Moreover, global climate changes cause more intense periods of precipitation than before (Intergovernmental Panel on Climate Change, 2007), increasing the risk of floods and overflows. In particular the combined sewer overflows can cause serious water pollution problems as untreated sewage is then released into the environment.

Sewers are operated using pumping stations to control the flows in the system. This operation is generally carried out manually and in a centralized way (Schütze et al., 2004). When storage facilities are present, they allow to store sewage during a storm. When the storm is over, sewage is pumped out of the storage facilities and sent to a wastewater treatment plant. An efficient operation of the pumping stations and an efficient use of the storage facilities can minimize the sewer overflows, the use of the water treatment plant, and consequently the uncontrolled release of pollutants.

The approach presented in this paper is in line with recent innovative work about modeling, simulation, and control of urban wastewater systems. Roughly, these approaches have a model-based framework, optimal control features, and realtime computation concerns in common (Schütze et al., 2004; Marinaki and Papageorgiou, 2005). The present work focuses on the interconnection between subsystems such as sewers, pumping stations, and water treatment plants, and is generally referred to as an integrated approach. Currently, these subsystems are considered as separate units and controlled locally without knowledge of the neighboring subsystems. Considering the whole system as one large-scale dynamical system in an integrated way allows to implement model-based control approaches (Schütze et al., 2004).

So-called model predictive control (MPC) strategies have in particular attracted the attention for over a decade due to their ability to handle explicitly economic objectives as well as operational constraints, see, e.g., (Gelormino and Ricker, 1994; Cembrano et al., 2004; Ocampo, 2007; Fiorelli and Schutz, 2009). In this paper we propose a novel nonlinear predictive control approach for improving the operation of sewer systems using a so-called pattern search optimization technique. Pattern search methods are very suitable here as the prediction model of the sewer system that is used in the controller is nondifferentiable and these methods do not require any explicit information about the gradient of the objective function or constraints. 
The paper is organized as follows. In Section 2 sewer systems are described and modeling issues are discussed. A case study based on the sewer system of Bogotá, Colombia, is introduced and modeled using the dedicated City Drain blockset (Achleitner et al., 2006) of Matlab-Simulink. In Section 3 the constrained predictive control problem of a sewer system is presented. The underlying nonlinear optimization problem is solved using a pattern search method in Section 4. Simulation results on the Bogotá case study are presented in Section 5 and conclusions and directions for future research are given in Section 6.

\section{SEWER SYSTEMS}

A sewer system is a drainage network composed of sewers, collectors, and surface or open channels that drain stormwater and urban sewage. A sewer system is in general composed of one or more watersheds (drainage bassin) the characteristics of which depend strongly on the topography of the area. In separated watersheds, a storm sewer is intended to transport only stormwater, surface runoff, or street drainage, whereas a sanitary sewer carries liquid and waterborne wastes from residences, buildings, industrial facilities, and institutions. In combined watersheds, sewers carry both stormwater and sewage. Since they mix with each other while flowing in the same pipe, stormwater is somehow contaminated with sewage in combined sewer systems.

To manage volumes and flows in the system, several elements are present, such as weirs, storage tanks, collectors, gates, valves, and pumping stations. A weir is a small dam that regulates the flow of water in an open channel. A weir can be used to allow sewage overflow into stormwater channels and consequently prevent sewage flooding. A storage tank is used to store excess rain in order to avoid overflows. In combined sewer systems such overflows are referred to as combined sewer overflows. A collector is a conduit that receives stormwater and/or sewage from lateral sewers or other branch conduits. Gates and valves are elements that essentially constrain or block the sewer and allow to regulate its flows. A pumping station is a facility including pumps and equipment to push sewage to water treatment plants. It is worth noting that a water treatment plant has a limited capacity (maximum flow).

Sewer systems can be considered as large-scale transportation networks. Under normal conditions, operators or local controllers act on the system with the aim to regulate locally volumes and flows in the network. Under particular conditions such as intense rainfall, volumes and flows grow rapidly, and flooding and combined sewer overflows may occur. Storage facilities and pumping stations may prevent or minimize such overflows.

\subsection{Bogotá sewer system}

The case study considered in this paper is based on a part of the sewer system of Bogotá, Colombia. The whole sewer network consists of approximately $5400 \mathrm{~km}$ of sanitary sewers and $2000 \mathrm{~km}$ of storm sewers. The case study consists of approximately $15 \%$ of the whole sewer system as depicted in Figure 1. It includes 25 watersheds of which 3 are combined watersheds. The watersheds are connected to collectors equipped with weirs. Each collector is equipped with a storage tank of $30 \mathrm{~m}^{3}$ with a maximum output flow rate of $0.02 \mathrm{~m}^{3} / \mathrm{s}$,

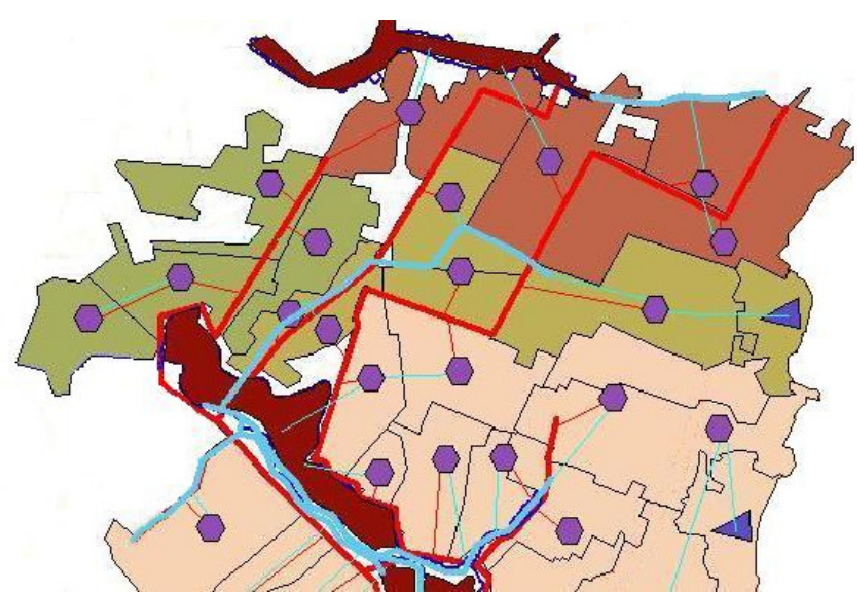

Fig. 1. Schematic representation of a part of the Bogotá sewer system with 22 separated watersheds (polygons) and 3 combined watersheds (triangles) interconnected by collectors (bold lines) and channels (thin lines).

and connected to a pump station equipped with a storage tank of $10 \mathrm{~m}^{3}$ that can pump sewage to a single water treatment plant with a maximum treatment capacity of $20 \mathrm{~m}^{3} / \mathrm{h}\left(0.0056 \mathrm{~m}^{3} / \mathrm{s}\right)$.

Due to frequent strong rainfall events, the Bogotá sewer system experiences regular sewer overflows and releases of contaminated water directly into the environment. Clearly the physical limitations in terms of maximum volumes and flows would not permit to prevent completely the overflows in the system, but an improvement in the coordination of the local control actions of storage tanks and pumping stations could help to reduce the overflows when such strong rainfall events occur. This is the purpose of the control scheme that is proposed in this paper. Therefore stormwater and sewage flows that enter into the system are considered as disturbances.

\subsection{Sewer system modeling using City Drain}

A model of the just described part of Bogotá sewer system has been implemented in City Drain and calibrated using real data by the Research Center in Environmental Engineering of Los Andes University ${ }^{1}$ jointly with the Water Distribution and Sewer System Company of Bogotá ${ }^{2}$ (Rodríguez et al., 2008). City Drain is an open source Matlab-Simulink blockset for integrated dynamic modeling of urban drainage systems (Achleitner et al., 2006). It incorporates a variety of models covering hydraulics, mass transport, processes for conversion of matter, etc. The user interface is block-oriented and blocks are connected to one another to provide information flow.

A sewer system model implemented using City Drain allows to easily compute the dynamic responses to stormwater and sewage flow profiles. In this paper, the City Drain blockset is used to build a prediction model for predictive control, i.e., this model will be used inside a control loop to predict the future behavior of the sewer system (and consequently its performance) and to choose the best action to apply with respect to given control objectives, see Section 3.

In City Drain, the following assumptions are made (Achleitner et al., 2006):

\footnotetext{
1 Centro de Investigación en Ingeniería Ambiental (CIIA).

2 Empresa de Acueducto y Alcantarillado de Bogotá (EAAB).
} 
- unsteady flows in conduits are one-dimensional;

- fluids are homogeneous and incompressible;

- pressure distribution is hydrostatic;

- the longitudinal axis of a conduit is approximated as a straight line;

- the bottom slope of a conduit does not change with the distance;

- the effects of scour and deposition are assumed to be negligible;

- friction can be described using the steady-state resistance laws.

Under those assumptions flows, sewers, weirs, storage tanks, and pumping station models are modeled as follows.

Flow in both sewers and conduits is described by the continuity and momentum equations of the well-known Saint-Venant equations (Schütze et al., 2002). The continuity equation represents the mass balance equation in the flow direction as:

$$
\frac{\partial a(x, t)}{\partial t}+\frac{\partial q(x, t)}{\partial x}=0,
$$

where $a$ is the cross-sectional area of the conduit $\left(\mathrm{m}^{2}\right), x$ is the spatial coordinate along the length of the conduit $(\mathrm{m}), t$ is the time (s), and $q$ is the flow rate $\left(\mathrm{m}^{3} / \mathrm{s}\right)$. The momentum equation is related to the momentum conservation and is given by:

$$
\begin{aligned}
& g a(x, t) \frac{\partial h(x, t)}{\partial x}+\frac{\partial q(x, t)}{\partial t} \\
& \quad-\sigma\left(2 \nu \frac{\partial a(x, t)}{\partial t}+\nu^{2} \frac{\partial a(x, t)}{\partial x}\right)+g a(x, t) s_{\mathrm{f}}(x, t)=0,
\end{aligned}
$$

where $h$ is the water level $(\mathrm{m}), \nu$ is the average velocity $(\mathrm{m} / \mathrm{s})$, $g$ is the gravitational constant $\left(\mathrm{m} / \mathrm{s}^{2}\right), \sigma$ is a constant depending on the flow depth, and $s_{\mathrm{f}}$ is the friction slope. The latter is determined using Manning's equation as:

$$
s_{\mathrm{f}}(x, t)=\frac{(n / 1.49)^{2}}{a(x, t) r^{\frac{4}{3}}} q(x, t)|\nu|,
$$

where $n$ is the coefficient of roughness and $r$ is the hydraulic radius $(\mathrm{m})$.

Weirs and storage tanks are modeled by a mass-conservation equation:

$$
\frac{\mathrm{d} v(t)}{\mathrm{d} t}=q_{\text {in }}(t)-q_{\text {out }}(t)-q_{\text {overflow }}(t)
$$

where $v$ is the volume stored in the tank $\left(\mathrm{m}^{3}\right), q_{\mathrm{in}}$ is the inflow rate $\left(\mathrm{m}^{3} / \mathrm{s}\right), q_{\text {out }}$ is the outflow rate $\left(\mathrm{m}^{3} / \mathrm{s}\right)$, and $q_{\text {overflow }}$ is the overflow $\left(\mathrm{m}^{3} / \mathrm{s}\right)$. The outflow rate $q_{\text {out }}$ is controllable within bounds $q_{\mathrm{out}}^{\min }$ and $q_{\mathrm{out}}^{\max }$, and is consequently considered as a bounded control input.

A pumping station consists of a storage tank and one or more pumping units. The state of the pump is controlled by a local on-off controller as illustrated in Figure 2. This discrete behavior can be described by a finite state automaton with 2 states. When the pump state $x_{\text {pump }}$ is off and the stored volume reaches the maximum threshold $v_{\text {pump }}^{\max }$ from below, the pump state becomes on. When the pump state is on and the stored volume reaches the minimum threshold $v_{\text {pump }}^{\min }$ from above, the pump state becomes off. In practice, the minimum threshold is a constant value that corresponds to a residual volume in order to avoid pump damage. The maximum threshold is then taken as a bounded control input.

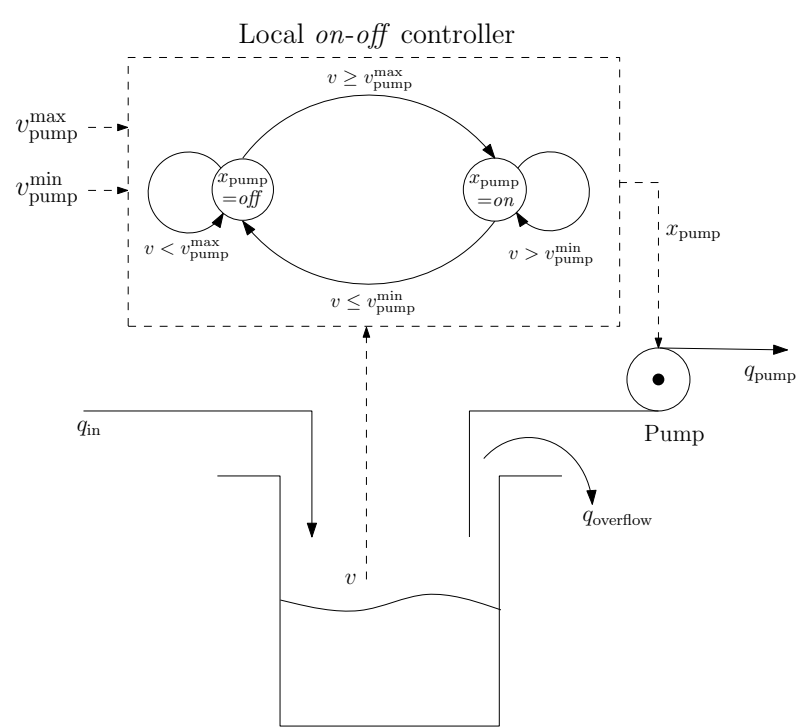

Fig. 2. Pumping station model.

In this implementation of the model a continuous state $\mathbf{x}_{\mathrm{c}}$ is defined to include the stored volumes and flow rates, a discrete state $\mathbf{x}_{\mathrm{d}}$ is defined to include the pump states (on/off), an output $\mathbf{y}$ is defined to include the pump, watershed and collector flow rates, the stored volumes, and the pump state, and an input $\mathbf{u}$ is defined to include the maximum thresholds of the pumps and outflow rates of the tanks.

\subsection{Prediction model}

This section details how the City Drain model can be used as a prediction model. Consider a time interval $\left[t_{0}, t_{\mathrm{f}}\right]$, i.e., the prediction interval. Given an initial continuous state $\mathbf{x}_{\mathrm{c}}\left(t_{0}\right) \in$ $\mathbb{R}^{n_{x_{\mathrm{c}}}}$, an initial discrete state $\mathbf{x}_{\mathrm{d}}\left(t_{0}\right) \in \mathbb{R}^{n_{x_{\mathrm{d}}}}$, and a collection of inputs $\mathbf{u}(t) \in \mathbb{R}^{n_{u}}$ over the full prediction interval, computing a prediction means solving an initial value problem and obtaining the outputs $\mathbf{y}(t) \in \mathbb{R}^{n_{y}}$, for $t \in\left[t_{0}, t_{\mathrm{f}}\right]$.

In a discrete-time control framework, control inputs are provided at discrete control sample steps $k_{\mathrm{c}}+i$, for $i=\{0,1, \ldots\}$, where $k_{\mathrm{c}}+i$ corresponds to continuous time $t_{0}+\left(k_{\mathrm{c}}+i\right) T_{\mathrm{c}}$ and $T_{\mathrm{c}}$ is the control sample time. A zero-order hold is used to maintain the control inputs constant between two control sample times, i.e., $\mathbf{u}(t)=\mathbf{u}\left(k_{\mathrm{c}}\right)$ for $t \in\left[t_{0}+k_{\mathrm{c}} T_{\mathrm{c}}, t_{0}+\left(k_{\mathrm{c}}+1\right) T_{\mathrm{c}}\right)$. Then a prediction model is given by a sequence of $N_{\mathrm{c}}$ inputs, $\tilde{\mathbf{u}}\left(k_{\mathrm{c}}\right)=\left[\mathbf{u}^{\mathrm{T}}\left(k_{\mathrm{c}}\right), \ldots, \mathbf{u}^{\mathrm{T}}\left(k_{\mathrm{c}}+N_{\mathrm{c}}-1\right)\right]^{\mathrm{T}}$, where $N_{\mathrm{c}}=$ $\left\lfloor\frac{t_{\mathrm{f}}-t_{0}}{T_{\mathrm{c}}}\right\rfloor+1$ is the number of control inputs over the prediction horizon $(\lfloor\tau\rfloor$ is the integer part of $\tau)$. Similarly, we assume that computing the output $\mathbf{y}$ for every $T_{\mathrm{c}}$ time units adequately represents the underlying continuous signals. The output sequence is then defined as $\tilde{\mathbf{y}}\left(k_{\mathrm{c}}\right)=\left[\mathbf{y}^{\mathrm{T}}\left(k_{\mathrm{c}}\right), \ldots, \mathbf{y}^{\mathrm{T}}\left(k_{\mathrm{c}}+N_{\mathrm{c}}-1\right)\right]^{\mathrm{T}}$.

The sewer system model built with City Drain does not contain algebraic loops nor implicit differential equations, and therefore without loss of generality, we can assume that the prediction model is given by the mapping

$$
\tilde{\mathbf{y}}\left(k_{\mathrm{c}}\right)=\mathcal{P}\left(\mathbf{x}_{\mathrm{c}}\left(t_{0}\right), \mathbf{x}_{\mathrm{d}}\left(t_{0}\right), \tilde{\mathbf{u}}\left(k_{\mathrm{c}}\right)\right),
$$

where $\mathcal{P}$ maps the initial continuous state $\mathbf{x}_{\mathrm{c}}\left(t_{0}\right)$ and discrete state $\mathbf{x}_{\mathrm{d}}\left(t_{0}\right)$ at time $t_{0}$, and the $N_{\mathrm{c}}$ inputs collected in $\tilde{\mathbf{u}}\left(k_{\mathrm{c}}\right)$ to the $N_{\mathrm{c}}$ outputs collected in $\tilde{\mathbf{y}}\left(k_{\mathrm{c}}\right)$. The prediction model $\mathcal{P}$ involves continuous-time dynamics in the form of nonlinear differential equations in combination with discrete-event dynam- 
ics in the form of discrete logic and if-then-else rules. Therefore the sewer system model is a nonlinear and nonsmooth dynamical system and consequently, computing numerical solutions, i.e., predictions, is a costly process.

\section{PREDICTIVE CONTROL OF SEWER SYSTEMS}

In model predictive control, the control actions are obtained at each control sample step $k_{\mathrm{c}}$ by solving an optimization problem that minimizes an objective function over a finite prediction horizon, subject to the evolution of the prediction model specified by the mapping $\mathcal{P}$ and operational constraints, e.g., on control inputs. The objective function and constraints for the case study are defined below.

\subsection{Objectives and constraints}

The following objectives are considered:

- Assure the use of the wastewater treatment plant at its full capacity while minimizing overflows that occur when the sum of the pump flows is larger than the maximum capacity of the treatment plant. This is done by minimizing for each prediction step $i$ (for $i=0, \ldots, N_{\mathrm{c}}-1$ ):

$$
\begin{aligned}
& J_{\mathrm{TP}}\left(\mathbf{y}\left(k_{\mathrm{c}}+i\right)\right)= \\
& w_{\mathrm{TP}}\left(\sum_{j=1}^{n_{\mathrm{pump}}} q_{\mathrm{pump}, j}\left(k_{\mathrm{c}}+i\right)-q_{\mathrm{TP}}^{\max }\right)^{2},
\end{aligned}
$$

where $q_{\text {pump }, j}$ is the output flow rate of the $j^{\text {th }}$ pump, $n_{\text {pump }}$ is the number of pumps, $q_{\mathrm{TP}}^{\max }$ is the maximal treatment capacity of the wastewater treatment plant, and $w_{\text {TP }}$ is a positive scalar weight.

- Reduce or avoid overflows of the watersheds (resp. storage tanks) of the sewer system by minimizing for each prediction step $i$ (for $i=0, \ldots, N_{\mathrm{c}}-1$ ) the term ${ }^{3}$ :

$$
J_{\text {overflow }}\left(\mathbf{y}\left(k_{\mathrm{c}}+i\right)\right)=\left\|\mathbf{q}_{\text {overflow }}\left(k_{\mathrm{c}}+i\right)\right\|_{\mathbf{W}_{\text {overflow }}}^{2},
$$

where qoverflow is the vector of overflow rates and $\mathbf{W}_{\text {overflow }}$ is a weight matrix. The $j^{\text {th }}$ component of qoverflow is the overflow of the $j^{\text {th }}$ watershed (resp. storage tank), defined as:

$$
\begin{aligned}
& q_{\text {overflow }, j}\left(k_{\mathrm{c}}+i\right)= \\
& \left\{\begin{array}{l}
0 \\
q_{\text {in }, j}\left(k_{\mathrm{c}}+i\right)-q_{\text {out }, j}\left(k_{\mathrm{c}}+i\right) \quad \text { otherwise, }
\end{array}\right.
\end{aligned}
$$

where $v_{j}$ is the volume stored in the watershed (resp. storage tank), $v_{j}^{\max }$ is the maximum volume that can be stored before overflow, $q_{\mathrm{in}, j}$ is the inflow rate, and $q_{\mathrm{out}, j}$ is the outflow rate.

- Minimize the economic cost when using the pumping stations since they can be used before rainfalls and/or after it has stopped falling, i.e., minimize for each prediction step $i$ (for $i=0, \ldots, N_{\mathrm{c}}-1$ ):

$$
J_{\text {pump }}\left(\mathbf{y}\left(k_{\mathrm{c}}+i\right)\right)=\sum_{j=1}^{n_{\text {pump }}} c_{j} x_{\text {pump }, j}\left(k_{\mathrm{c}}+i\right),
$$

where $x_{\text {pump }, j}$ is the discrete state of the $j^{\text {th }}$ pump, i.e., $x_{\text {pump }, j}=1$ when the pump is working (state on) and $x_{\text {pump }, j}=0$ otherwise (state off). The constant $c_{j}$ is

\footnotetext{
$3\|\mathbf{x}\|_{\mathbf{W}}$ is the weighted 2-norm of $\mathbf{x}$, i.e., $\mathbf{x}^{\mathrm{T}} \mathbf{W} \mathbf{x}$ with positive-semidefinite matrix $\mathbf{W}$.
}

positive and represents the economic cost of using the $j^{\text {th }}$ pump.

The combined control objectives over the prediction horizon are now defined as:

$$
\begin{aligned}
& J\left(\tilde{\mathbf{u}}\left(k_{\mathrm{c}}\right), \tilde{\mathbf{y}}\left(k_{\mathrm{c}}\right)\right)=\sum_{i=0}^{N_{\mathrm{c}}-1}\left(J_{\mathrm{TP}}\left(\mathbf{y}\left(k_{\mathrm{c}}+i\right)\right)\right. \\
& \left.\quad+J_{\text {overflow }}\left(\mathbf{y}\left(k_{\mathrm{c}}+i\right)\right)+J_{\text {pump }}\left(\mathbf{y}\left(k_{\mathrm{c}}+i\right)\right)\right) .
\end{aligned}
$$

The bounds on the control inputs $\mathbf{u}$, e.g., minimum and maximum thresholds $v_{\text {pump }}^{\min }$ and $v_{\text {pump }}^{\max }$ for the local controller of the pumps, and minimum and maximum output flow rates $q_{\text {out }}^{\min }$ and $q_{\text {out }}^{\max }$ of the storage tanks, are taken into account in the form of inequality constraints:

for $i=0, \ldots, N_{\mathrm{c}}-1$.

\subsection{MPC optimization problem}

The MPC problem can now be formulated as an optimization problem that has to be solved at each control step, given current states $\mathbf{x}_{\mathrm{c}}\left(t_{0}\right), \mathbf{x}_{\mathrm{d}}\left(t_{0}\right)$, and $\mathbf{u}\left(k_{\mathrm{c}}-1\right)$ at $t_{0}$ :

$$
\min _{\tilde{\mathbf{u}}\left(k_{\mathrm{c}}\right), \tilde{\mathbf{y}}\left(k_{\mathrm{c}}\right)} J\left(\tilde{\mathbf{u}}\left(k_{\mathrm{c}}\right), \tilde{\mathbf{y}}\left(k_{\mathrm{c}}\right)\right)
$$

subject to

$$
\begin{gathered}
\tilde{\mathbf{y}}\left(k_{\mathrm{c}}\right)=\mathcal{P}\left(\mathbf{x}_{\mathrm{c}}\left(t_{0}\right), \mathbf{x}_{\mathrm{d}}\left(t_{0}\right), \tilde{\mathbf{u}}_{\mathrm{c}}\left(k_{\mathrm{c}}\right)\right) \\
\mathbf{u}_{\text {lower }} \leq \mathbf{u}\left(k_{\mathrm{c}}\right) \leq \mathbf{u}_{\text {upper }} \\
\vdots \\
\mathbf{u}_{\text {lower }} \leq \mathbf{u}\left(k_{\mathrm{c}}+N_{\mathrm{c}}-1\right) \leq \mathbf{u}_{\text {upper }} .
\end{gathered}
$$

By substituting the prediction model (13) into the objective function (12) an optimization problem with a nonlinear, nondifferentiable objective function and simple box constraints is obtained.

\section{PATTERN SEARCH METHODS FOR NONLINEAR OPTIMIZATION}

In the MPC problem defined above (after the substitution of the prediction model) evaluating the objective function is expensive due to the due to the evaluation of the prediction model which involves a Simulink simulation. In practice, the computation time is limited and within the available computation time a solution that is as good as possible has to be determined. Many nonlinear optimization methods rely on gradient and Hessian information (Nocedal and Wright, 1999). Solvers that use this first-order or second-order information will therefore perform numerical approximation of the gradient and/or Hessian, involving numerous objective function evaluations. In addition, due to the non-smoothness of the problem there are many local minima in which this type of solvers typically quickly can get stuck.

Instead of using gradient or Hessian-based solvers, we propose to use a so-called direct-search optimization method, which does not explicitly require gradient and Hessian information (Wright, 1996). The only property that this method requires is that the values of the objective function can be ranked. This feature together with the feature that direct-search methods are suitable for non-smooth problems, make that such a method is suitable for solving the control problem at hand. 
In particular, we propose to use the direct-search method pattern search (Lewis et al., 2000) due to its straightforward implementation and its ability to yield good solutions, even for objective functions with many local minima.

Pattern search works in an iterative way in which a solution $\mathbf{x}_{s}$ at iteration $s$ is replaced by a new solution $\mathbf{x}^{+}$only if $f\left(\mathbf{x}^{+}\right)<f\left(\mathbf{x}_{s}\right)$. In addition, the new solution $\mathbf{x}^{+}$is selected from a finite set of candidate solutions $\mathcal{M}_{s}$ that is updated at each iteration. An iteration of pattern search is summarized in the following steps (Lewis et al., 2000):

- A mesh $\mathcal{M}_{s}$ around the current solution $\mathbf{x}_{s}$ is constructed, consisting of a discrete set of candidate solutions in $\mathbb{R}^{n}$ in which the algorithm searches for a new solution. The coarseness of the mesh is determined by the mesh size $\Delta_{s} \in \mathbb{R}^{+}$.

- The mesh $\mathcal{M}_{s}$ is explored in one or two phases:

- In the search phase any strategy can be used to find a solution $\mathbf{x}^{+} \in \mathcal{M}_{s}$ for which $f\left(\mathbf{x}^{+}\right)<f\left(\mathbf{x}_{s}\right)$, as long as a finite number of points is considered. If a solution $\mathbf{x}^{+}$is found, the search was successful and the next phase is not invoked.

- In the polling phase a new solution $\mathbf{x}^{+}$for which $f\left(\mathbf{x}^{+}\right)<f\left(\mathbf{x}_{s}\right)$ is searched for in a subset of solutions in $\mathcal{M}_{s}$, consisting of those solutions that are in the direct neighborhood of the current solution $\mathbf{x}_{s}$. This neighborhood is defined through a set of vectors called a pattern and the current solution. If a solution $\mathbf{x}^{+}$is found in this neighborhood then the polling phase was successful.

- If either of the phases was successful, then $\mathbf{x}_{s+1}:=\mathbf{x}^{+}$, the coarseness of the mesh is set to $\Delta_{s+1}=\epsilon \Delta_{s}$, with expansion factor $\epsilon>1$, and the next iteration starts. If $\mathbf{x}^{+}$was not found, then $\mathbf{x}_{s+1}:=\mathbf{x}_{s}$, the coarseness of the mesh is set to $\Delta_{s+1}=\gamma \Delta_{s}$, with a contraction factor $\gamma \in(0,1)$, and the next iteration starts.

The iterations continue until a stopping condition is satisfied, e.g., the mesh size is less than a given tolerance, the total number of objective function evaluations reaches a given maximum, or the distance between the point found at one successful poll and the point at the next successful poll is less than a given tolerance.

In our simulations in the next section we use the pattern search implementation as included in the Genetic Algorithm and Direct Search Toolbox of Matlab v. 2.3 (R2008a) (The Matworks, 2008).

\section{SIMULATION RESULTS}

This section presents the simulation results obtained with the Bogotá case study introduced in Section 2.1. The City Drain model is used both for representing the system to be controlled and as a prediction model in the model predictive controller. The dimension of the continuous state $\mathbf{x}_{\mathrm{c}}$ is 59 (31 volumes and 25 flow rates). The dimension of the discrete state is 3 (pump states) and the dimension of the control input is 6 (maximum threshold of local controller of the pumps and output flow rate of the storage tanks).

Rainfall and sewage flow rates are considered as measured disturbances (3 stormwater and 3 sewage input flow rates). Typically, rainfall and sewage predictions until up to $5 \mathrm{~h}$ into the future are considered accurate. The average settling-time of

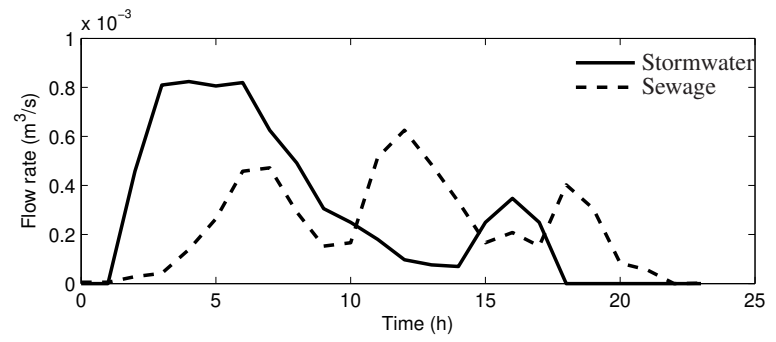

Fig. 3. Stormwater (solid line) and sewage (dashed line) flow rates profiles.
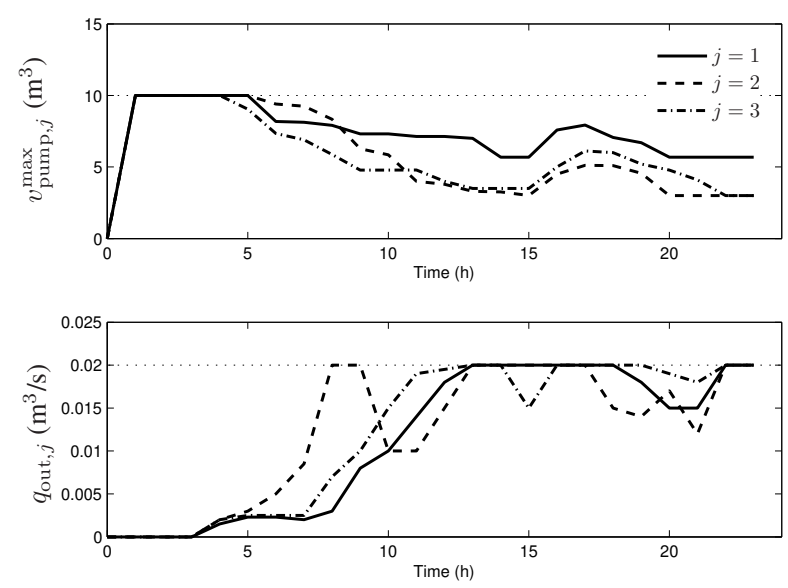

Fig. 4. Maximum thresholds of the local controller of the pumps (top) and the output flow rates of the storage tanks (bottom) with corresponding bounds (dotted lines).

the system is around $1 \mathrm{~h}$. Consequently, the control step $T_{\mathrm{c}}$ is selected equal to $1 \mathrm{~h}$. The prediction horizon is $5 \mathrm{~h}$, i.e., $N_{\mathrm{c}}=5$ and the simulation time is $24 \mathrm{~h}$, starting at midnight.

Hereafter, a scenario in which a strong rainfall event occurs in the morning is used to illustrate the operation of the proposed approach. This scenario arises from statistical studies based on historical data collected between 2006 and 2008, and illustrates a typical case (Rodríguez et al., 2008). Figure 3 shows the total stormwater and sewage flow rate profiles used over the $24 \mathrm{~h}$ simulation time.

The MPC controller is tuned with the following values: $w_{\mathrm{TP}}=$ $10^{3}, \mathbf{W}_{\text {overflow }}$ is a diagonal matrix with all diagonal elements equal to $10^{3}$, and $c_{1}=c_{2}=c_{3}=1$. The control inputs should stay within the following bounds: $\mathbf{u}_{\text {lower }}=\left[\begin{array}{llllll}0 & 0 & 0 & 0 & 0 & 0\end{array}\right]^{\mathrm{T}}$ and $\mathbf{u}_{\text {upper }}=\left[\begin{array}{llllll}30 & 30 & 30 & 0.02 & 0.02 & 0.02\end{array}\right]^{\mathrm{T}}$.

Figure 4 shows the maximum thresholds $v_{\text {pump }}^{\max }$ of the local controller of the 3 pumps and the output flow rates $q_{\text {out }}$ of the storage tanks with respect to time, as well as corresponding bounds. Figure 5 shows the evolution of the stored volumes in the tanks over the simulation. Figure 6 shows the summed overflows over the simulation, both when the proposed scheme is used and when operating in manual control (in which the maximum threshold value of the local controller of each pump is equal to the maximum capacity of the storage tank and storage tank of collectors have the maximum output flow rate). It is observed that the summed overflow under the proposed MPC scheme is reduced by $40 \%$ with respect to the summed overflow in manual operation. Moreover the maximum treatment 

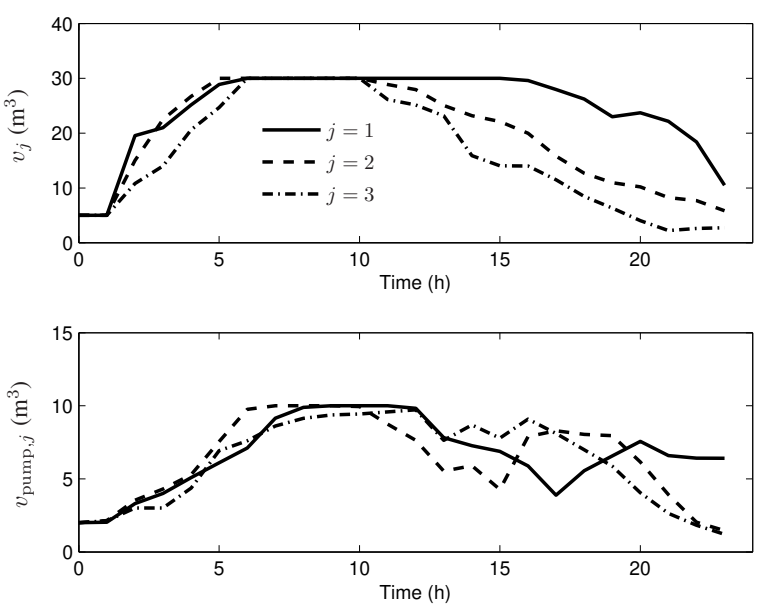

Fig. 5. Stored volumes in storage tanks (top) and tanks of pumping stations (bottom).

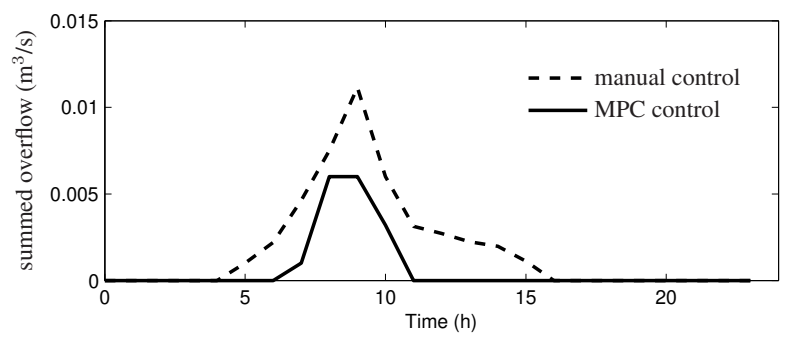

Fig. 6. Summed overflows for manual control and MPC control: the summed overflow is reduced by $40 \%$.

capacity of the water treatment plant $\left(0.0056 \mathrm{~m}^{3} / \mathrm{s}\right)$ is respected with the MPC scheme.

These results illustrate how the proposed MPC scheme is able to successfully prevent overflows of untreated wastewater.

\section{CONCLUSIONS AND FUTURE WORK}

This paper presents a model predictive control (MPC) strategy based on a pattern search method for sewer system operation. The MPC controller uses an internal model of the system to predict its future behavior and to choose adequately the best actions to apply to minimize the consequences of strong rainfall events, i.e., overflows, and to improve the operation. The prediction model has been built with the City Drain blockset of Matlab-Simulink. The prediction model is non-differentiable and a pattern search algorithm is used to solve the underlying optimization problem that arises from the MPC strategy. The results show the capabilities of the proposed approach applied to the operation of a sewer system.

Future research will focus on further assessing the performance of the proposed scheme in particular, taking into account practical time constraints. Moreover, robustness of the scheme will also be investigated by explicitly considering uncertainty in the disturbance profiles (rainfall and sewage). Furthermore, distributed implementations of the scheme will be considered in a distributed MPC setup in which the different parts of the city will have dedicated MPC controllers, which via coordination locally choose actions that are system-wide optimal.
The authors would like to thank M.A. Díaz-Granados, J.P. Rodríguez, and F. Avila of the CIIA of Los Andes University for making available the City Drain model used in this work. This research is financially supported by the BSIK project "Next Generation Infrastructures (NGI)", the Delft Research Center Next Generation Infrastructures and the European STREP project "Hierarchical and distributed model predictive control (HD-MPC)".

\section{REFERENCES}

Achleitner, S., Möderl, M., and Rauch, W. (2006). CITY DRAIN: An open source approach for simulation of integrated urban drainage systems. Environmental Modeling \& Software, 22(8), 1184-1195.

Cembrano, C., Quevedo, J., Salamero, M., Puig, V., Figueras, J., and Martí, J. (2004). Optimal control of urban drainage systems. A case study. Control Engineering Practice, 12(1), $1-9$.

Fiorelli, D. and Schutz, G. (2009). Real-time control of a sewer network using a multi-goal objective function. In Proceedings of the 17th Mediterranean Conference on Control \& Automation, 676-681. Thessaloniki, Greece.

Gelormino, M. and Ricker, N. (1994). Model predictive control of a combined sewer system. International Journal of Control, 59(3), 793-816.

Hendricks, D. (2005). Water Treatment Unit Processes: Physical and Chemical. Taylor \& Francis, London, UK.

Intergovernmental Panel on Climate Change (2007). Climate Change 2007 - The Physical Science Basis. Cambridge University Press, New York, New York.

Lewis, R.M., Torczon, V., and Trosset, M.W. (2000). Direct search methods: then and now. Journal of Computational and Applied Mathematics, 124(1-2), 191-207.

Marinaki, M. and Papageorgiou, M. (2005). Optimal Real-time Control of Sewer Networks. Springer-Verlag, London, UK.

Nocedal, J. and Wright, S. (1999). Numerical Optimization. Springer Series in Operations Research. Springer-Verlag, New York, New York.

Ocampo, C. (2007). Model Predictive Control of Complex Systems including Fault Tolerance Capabilities: Application to Sewer Networks. Ph.D. thesis, Technical University of Catalonia, Barcelona, Spain.

Rodríguez, J., Díaz-Granados, M.A., Camacho, L., Raciny, I., Maksimovic, C., and McIntyre, N. (2008). Bogota's urban drainage system: context, research activities and perspectives. In Proceedings of the 10th National Hydrology Symposium, British Hydrological Society. Exeter, United Kingdom.

Schütze, M., Butler, D., and Beck, M. (2002). Modeling, Simulation and Control of Urban Wastewater Systems. SpringerVerlag, London, UK.

Schütze, M., Campisano, A., Colas, H., Schilling, W., and Vanrolleghem, P. (2004). Real-time control of urban wastewater systems - Where do we stand today? Journal of Hydrology, 299(3), 335-348.

The Matworks (2008). Genetic Algorithm and Direct Search Toolbox User's Guide. The Matworks.

Wright, M.H. (1996). Direct search methods: Once scorned, now respectable. In D.F. Griffiths and G.A. Watson (eds.), Numerical Analysis 1995, 191-208. Addison Wesley, Harlow, UK. 